\title{
On Accretion of Nanosize Carbon Spheres
}

\author{
Z. C. Kang and Z. L. Wang* \\ School of Material Science and Engineering, Georgia Institute of Technology, Atlanta, Georgia 30332-0245 \\ Received: December 4, $1995^{\otimes}$
}

\begin{abstract}
In this paper, the high chemical activity of the newly synthesized nanosize carbon spheres is demonstrated. The carbon spheres can be accreted by a treatment in acetone. The mechanism for the accretion is attributed to the combination of the hydrogen and oxygen atoms belonging to the acetone molecules with the adsorbed hydrogen and/or oxygen atoms at the edges of the open graphitic flakes distributed at the surface of the carbon spheres. The high chemical activity of the carbon spheres can have important applications in catalysis. The accretion of the carbon spheres may significantly improve the strength of composite materials made using the spheres.
\end{abstract}

The bonding in diamond is dominated by the $\mathrm{sp}^{3}$ electronic configuration, the four valence electrons of a carbon atom are all in the three-dimensional tetrahedral oriented $\mathrm{sp}^{3}$ hybrid orbital, forming strong $\sigma$ bonding with the adjacent atoms. ${ }^{1}$ For graphite, the 3 -fold coordinated $\mathrm{sp}^{2}$ electronic configuration makes the three out of four valence electrons in a twodimensional trigonally oriented $\mathrm{sp}^{2}$ hybrid orbital, forming strong intralayer $\sigma$ bonding within the hexagonal carbon rings; the fourth electron is in the p $\pi$ orbital which is perpendicular to the hexagonal network, forming a weakly unsaturated $\pi$ bonding with adjacent layers. The discover of $\mathrm{C}_{60}{ }^{2}$ has made a greatly impact on the understanding of carbon bonding. For a $\mathrm{C}_{60}, 12$ pentagonal carbon rings must be introduced to form a close cage structure with 20 hexagonal carbon rings. Each atom in $\mathrm{C}_{60}$ has three adjacent neighbors although the atoms may form pentagonal carbon rings. Thus, the nearest-neighbor distribution in $\mathrm{C}_{60}$ is approximately equivalent to the atom distribution in a hexagonal graphite layer, and the electronic configuration can still be described by the $\mathrm{sp}^{2}$ hybrid orbital although some distortion may exist due to the atom distribution in the $\mathrm{C}_{60}$ cage. $^{1}$ The fourth unsaturated $\pi$-bonding electron would like to form a pair with an electron from another $\mathrm{C}_{60}$, forming the weakly bonded $\mathrm{C}_{60}$ crystals. Thus, $\mathrm{C}_{60}$ can function as an "element" that can be combined with other metallic elements, which can donate electrons, to form crystalline structure. ${ }^{3}$ Moreover, the $\mathrm{C}_{60}$ cage is unlikely to be opened by an external electron due to the weakness of the unsaturated $\pi$ bonding normal to the surface. Therefore, $\mathrm{C}_{60}$ is considered to be chemically inert.

Carbon spheres with closed shell structure are expected to have analogous chemical characteristics as $\mathrm{C}_{60}$. However, the situation could be very different if the graphitic layers composing the carbon spheres are unclosed, leaving many dangling bonds at the open sites. The study of chemical activity of carbon spheres, to our knowledge, has not been reported because of the lack of techniques for synthesized nanosize carbon spheres. Recently, we introduced a mixed-valence oxides catalytic carbonization (MVOCC) process for synthesizing monodispersive carbon spheres (or tubes) in macroscopic quantities at low cost and with high-purity product. ${ }^{4,5}$ The spheres are composed of graphitic flakes and are believed to exhibit many dangling bonds at the surfaces. In this paper, the accretion of the newly

* To whom the correspondence should be sent. E-mail: zhong.wang@ mse.gatech.edu.

${ }^{\otimes}$ Abstract published in Advance ACS Abstracts, March 1, 1996.
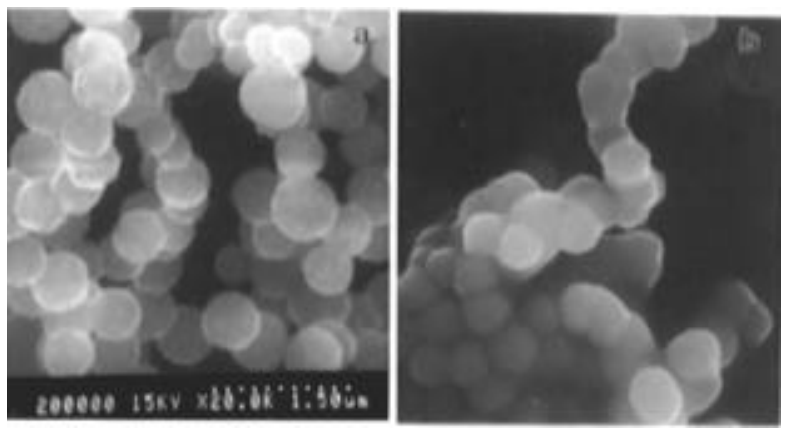

Figure 1. SEM images of the carbon spheres (a) before and (b) after being treated in acetone for $10 \mathrm{~min}$, showing the accretion of the spheres after the treatment. The SEM images were recorded at $15 \mathrm{kV}$ using a Hitachi S800.

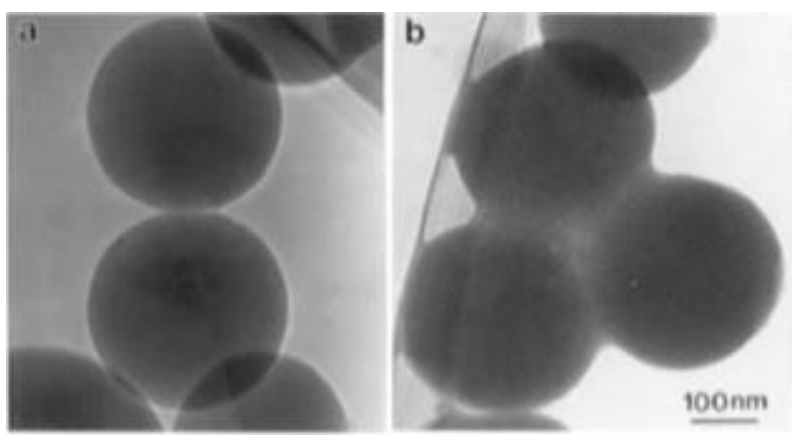

Figure 2. TEM images showing the (a) double and (b) triple accretion of the carbon spheres.

synthesized carbon spheres is demonstrated to show their high chemical activity. The accretion mechanism is discussed using a surface adsorption model.

To show the high chemical activity, the morphological and structural evaluations of the spheres are determined before and after being treated ultrasonically for $10 \mathrm{~min}$ in acetone. Scanning electron microscopy (SEM) and transmission electron microscopy (TEM) were used to characterize the accretion among the spheres. Figure 1, parts a and b, show two SEM images of the carbon spheres before and after being treated in acetone, respectively. The untreated spheres show clear shapes with well-dispersed distribution (Figure 1a), while the spheres treated in acetone are tightly accreted. Fine details can be seen on the surfaces of the spheres, corresponding to the unclosed graphitic flakes on the surface. The carbon atoms at the edge 

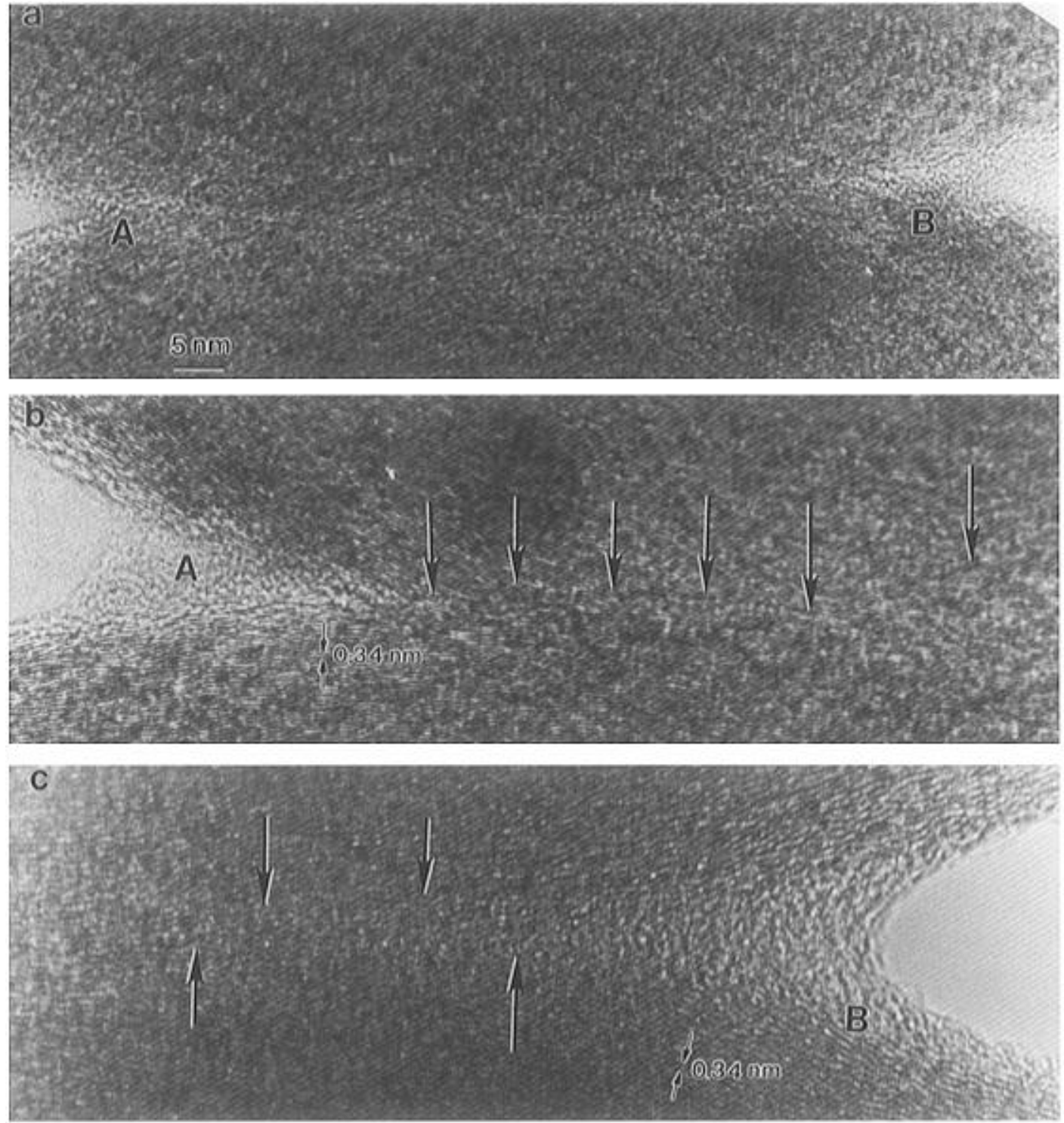

Figure 3. (a) High-magnification TEM images of the double accreted spheres in Figure 2a, showing the interfacial microstructure at the (b) leftand (c) right-hand sides of the accreted region. Both spheres are in contact, and there is an amorphous layer adjacent to the interface. TEM observations were performed using a Hitachi HF-2000 $(200 \mathrm{kV})$ equipped with a field emission gun.

of the curling graphitic flakes must have dangling bonds with unpaired $\mathrm{sp}^{2}$ orbital. These sites are expected to be chemically active.

Various accretion geometry can be produced by a treatment in acetone. Figure 2 shows the double and triple accreted carbon spheres. The two spheres in Figure $2 \mathrm{a}$ are just attached on each other, and the three spheres in Figure $2 b$ are tightly accreted. The intersphere is filled with "glue" type of material that has been identified as carbon using energy-dispersive X-ray spectroscopy. Figure 3 gives high-resolution TEM images of the accreted interfacial region of the two spheres shown in Figure $2 \mathrm{a}$, and the corresponding regions are marked with $\mathrm{A}$ and $\mathrm{B}$. The accreted region shows clearly the graphitic layered structure with interlayer distance approximately $0.34 \mathrm{~nm}$, corresponding to the (002) interplanar distance of graphite. The interface of the two spheres can be seen in the region indicated by arrowheads in Figure 3b,c. The graphitic flakes can be as small as $1 \mathrm{~nm}$. Some broken graphitic layers are visible at region B in Figure 3c, where the two spheres are separated by a thin amorphous layer.

To understand the mechanism for the formation of the accreted structure, we first review the structural model of these spheres as reported in detail elsewhere. ${ }^{6}$ Figure 4 shows a high-

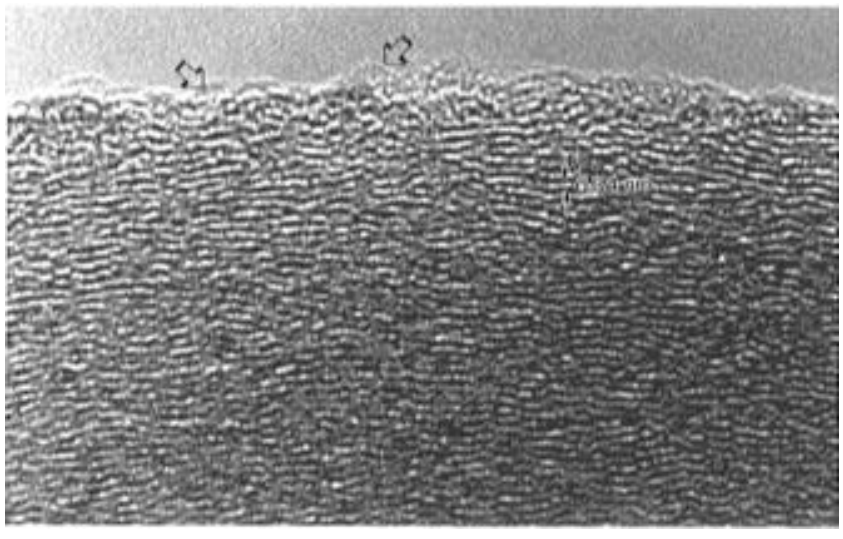

Figure 4. High-resolution TEM image near the surface of a carbon sphere before the treatment in acetone, showing unclosed graphitic flakes at the surface. The fine details seen at the surface are possible high-density chemical reaction sites, as indicated by arrowheads.

resolution TEM image of a carbon sphere before treated in acetone. The graphitic flakes composing the spheres are apparently unclosed. The sphere is believed to be nucleated from a pentagon carbon ring followed by a spiral shell growth, ${ }^{7}$ 


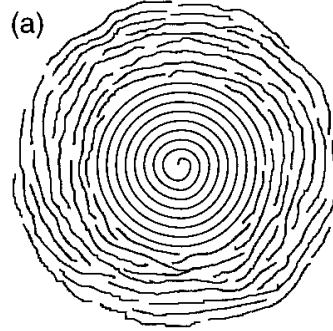

(b) Hexagon (zero curvature)

(c) Pentagon (positive curvature)

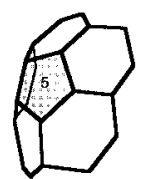

Carbon sphere

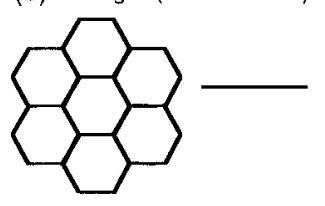

(d) Heptagon (negative curvature)

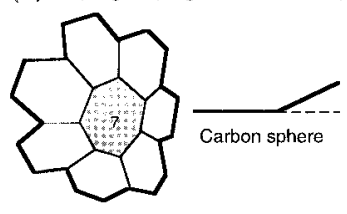

Figure 5. (a) Schematic structural model of the carbon sphere composed with graphitic flakes. The (b) hexagonal, (c) pentagonal, and (d) heptagonal carbon rings used to assemble the graphitic flakes. The pentagonal and heptagonal carbon rings introduce changes in the curvature of the graphitic flake to accommodate the geometry of the interior layers.

(a) Acetone

(b)<smiles>CC(C)=O</smiles>

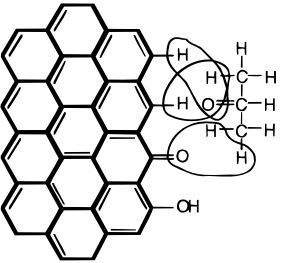

(c)

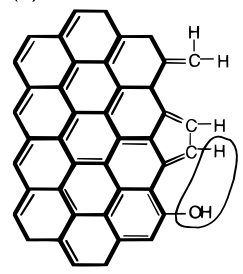

Figure 6. (a) Chemical bonding in an acetone molecule. (b, c) Reaction process of an acetone molecule with an open edge of a hexagonal graphitic flake.

as shown in Figure 5a. When the sphere grows larger, graphitic flakes of atom thickness are nucleated on the surface due to the arrival of the paired pentagonal-heptagonal carbon rings as described below. The pentagonal, hexagonal, and heptagonal carbon rings are required to accommodate the curvature of the carbon sphere. A flat graphite layer is composed of the hexagonal carbon rings (Figure 5b). The pentagonal carbon ring (Figure 5c) causes the hexagonal network to curve inward (with $+60^{\circ}$ disclinations), forming a surface with positive curvature. The heptagonal carbon ring forces the hexagonal lattice to be curved outward (with $-60^{\circ}$ disclinations, Figure 5d). The geometrical combinations of these carbon rings can make all the structural models observed experimentally. ${ }^{6}$ Therefore, the spheres are composed of curling graphitic flakes with random twisting, and these flakes are not closed shells but small-size waving flakes, ranging in sizes from $1-10 \mathrm{~nm}$ (Figure 4). The edges of the graphitic flakes are ideal sites for absorbing hydrogen and oxygen, resulting in the chemical reaction with acetone.

Chemically, an acetone molecular (Figure 6a) has a strong electrostatic polarity and the oxygen atom has double bonds with the carbon atom. The polarity is along the $\mathrm{C}-\mathrm{O}$ chain since the six hydrogen atoms and three carbon atoms are distributed with mirror symmetry. The oxygen atom is nega-

tively charged and the carbon atom is positively charged, as indicated in Figure 6a. The acetone molecule will be quickly attracted once it meets the edge of a graphitic flake, where the unpaired $\mathrm{sp}^{2}$ orbital is usually balanced by the adsorbed hydrogen, oxygen, and/or $\mathrm{OH}$ (Figure 6b). ${ }^{8}$ The strength of the polarized $\mathrm{C}-\mathrm{H}$ bonding is weaker than that of $\mathrm{C}-\mathrm{O}$. Thus, the acetone molecule might tend to approach the hydrogen atom sites. The combination of an adsorbed hydrogen atom with a hydrogen atom and the oxygen atom of the acetone molecule produces a water molecule $\mathrm{H}_{2} \mathrm{O}$, which will be evaporated, resulting in the combination of the carbon atoms with the edge of the graphitic flake. The surface adsorbed oxygen can also combine with two hydrogen atoms belonging to the acetone molecule to form $\mathrm{H}_{2} \mathrm{O}$. The active energy for these reactions may be provided by the ultrasonic wave. The carbon atoms from the acetone molecules are likely to be packed in amorphous (see Figure $3 b$ ) because of the lack of activation energy at room temperature, consistent with the observation shown in Figure 3. High-temperature annealing may graphitize the structure. When another sphere approaches the acetone molecule from the right-hand side, the hydrogen atoms belonging to the acetone molecule (Figure 6c) may combine with the oxygen atoms adsorbed at the edge of a graphite flake belonging to the other sphere, the evaporation of $\mathrm{H}_{2} \mathrm{O}$ leaves the carbon atoms, which tend to connect the two spheres, resulting in the accretion effect. Analogous chemical processes can occur if the edges of the graphitic flakes have pentagonal and/or heptagonal carbon rings. In general, no accretion by acetone is expected if the carbon spheres are composed of closed graphitic shells

The rate of carbon sphere accretion in acetone is much faster than that in ethanol. This is probably because the ethanol molecule has much weaker electrostatic polarity than that of acetone, and it may not be strongly attracted by the graphitic flakes of the carbon sphere.

In conclusion, high chemical activity of the newly synthesized carbon spheres has been demonstrated. The accretion of the spheres is believed to be directly related to the hydrogen and/ or oxygen atoms adsorbed at the edges of the open graphitic flakes. The high surface chemical reactivity of the carbon spheres clearly indicates that these spheres can be used as a support of catalyst. The accretion property of the carbon spheres may have important application to improve the strength of composite materials made using the spheres.

\section{References and Notes}

(1) Roberson, J. Prog. Solid State Chem. 1991, 21, 199.

(2) Kroto, H. W.; Heath, J. R.; O’Brien, S. C.; Curl., R. F.; Smalley, R. E. Nature 1985, 318, 162.

(3) Krätschmer, W., Lamb, L. D.; Fostiropoulos, K.; Huffman, D. Nature 1990, 347, 354. Zhu, Q.; Cox, D. E.; Fischer, J. E.; Kniaz, K.; McGhie, A. R.; Zhou, O. Nature 1992, 355, 712.

(4) Kang, Z. C.; Wang, Z. L. US patent in pending (Georgia Institute of Technology), 1996.

(5) Kang, Z. C.; Wang, Z. L. Science, submitted; Philos. Mag. B, in press.

(6) Wang, Z. L.; Kang, Z. C. Phys. Rev. Lett., submitted.

(7) Kroto, H. W.; McKay, K. Nature 1988, 331, 328.

(8) Fabish, T. J.; Schleifer, Carbon 1984, 22, 19. 